\title{
Amorphous Gallium Phosphate Thin Films: Ga and O K-Edge Absorption Spectroscopy
}

\author{
F. Tourtin, P. Armand, A. Ibanez*, G. Tourillon** and E. Philippot \\ LPMC, UMR 5617, Case 003, Place E. Bataillon, 34095 Montpellier cedex 05, France \\ * Laboratoire de Cristallographie, CNRS, 25 avenue des Martyrs, BP. 166, 38042 Grenoble cedex 09, \\ France \\ ** LURE, Bât. 209D, Centre Universitaire Paris-Sud, 91405 Orsay cedex, France
}

\begin{abstract}
Amorphous dielectric gallium phosphate thin films of various compositions are obtained on silicon substrate by the "pyrosol" process. We report, in this paper, the results of a structural characterization of these deposits. The atomic surroundings of gallium atoms have been determined by a Ga K-edge X-ray absorption study. Oxygen K-edge XANES measurements, completed by $O 1 s$ core-level XPS data collection, were carried out in order to probe the oxygen environment. In phosphorous-rich thin films, gallium atoms are in mixed surroundings constituted by both tetrahedral $\mathrm{GaO}_{4}$ and octahedral $\mathrm{GaO}_{6}$ sites. The oxygen atoms are divalent, and we pointed out the coexistence of Ga-O.P and $\mathrm{P}-\mathrm{O}-\mathrm{P}$ linkages. In gallium-enriched deposits, $\mathrm{GaO}_{6}$ sites are predominant. The oxygen atoms are mainly dicoordinated to gallium ones, while some are non-bridging.
\end{abstract}

\section{INTRODUCTION}

Native oxyde layers are usually assumed to be poor candidates to passivate III-V semiconductor compounds, such as GaAs or InP. Indeed, there are generally inhomogeneous and give insulator-semiconductor interfaces with poor electrical properties. To overcome these problems, one solution would be to replace the thermal growth by an insulating deposit of high dielectric properties at low temperature. Since $\mathrm{M}^{\mathrm{III}} \mathrm{V} \mathrm{O}_{4}$ materials, such as $\alpha$-quartz gallium orthophosphate $\left(\mathrm{GaPO}_{4}\right)$ and berlinite $\left(\mathrm{AlPO}_{4}\right)$ exhibit interesting piezoelectric and dielectric properties similar to or better than those of $\alpha$-quartz $\mathrm{SiO}_{2}$ [1], we set about developing gallium phosphate thin films by the "pyrosol" process. This process [2] allows to obtain good dielectric deposits at low temperatures $\left(<500^{\circ} \mathrm{C}\right)$. We first undertaken the elaboration of gallium phosphate films on silicon substrates [3] before to use GaAs wafers. All the synthesized layers on Si substrate are amorphous and a large range of chemical compositions in terms of experimental conditions can be observed [3]. These compositions are expressed by the ratio Ga/P of the atomic percentages of gallium and phosphorus as determined by electron probe microanalysis. Oxygen excess as compared to stoichiometric $\mathrm{GaPO}_{4}$, is expressed by the ratio $\mathrm{O}_{\mathrm{th}} / \mathrm{O}_{\text {exp. }}$.

This paper reports results coming out from structural characterizations of both the $\mathrm{Ga}$ and $\mathrm{O}$ surroundings in raw gallium phosphate thin films deposited on silicon substrates.

\section{GALLIUM ENVIRONMENT}

In order to characterize the local atomic environment around the gallium atoms, we recorded $\mathrm{Ga} \mathrm{K}$-edge $(10367 \mathrm{eV}) \mathrm{X}$-ray absorption spectra for thin films of various compositions and for several crystalline compounds of known structure: $\alpha G^{2} \mathrm{O}_{4}$ (quartz-like structure, where $\mathrm{Ga}$ atoms are in tetrahedra sites $\mathrm{GaO}_{4}[6]$ ), $\mathrm{\beta Ga}_{2} \mathrm{O}_{3}(\mathrm{Ga}$ are located in both tetrahedral and octahedral sites [5]) and $\mathrm{GaOOH}$ (Ga exhibit only octahedral $\mathrm{GaO}_{6}$ sites [7)].

Figure 1 brings together the normalized XANES curves of thin films enriched in phosphorus and those of $\alpha_{\mathrm{GaPO}}$ and $\mathrm{GaOOH}$. The P-rich $(\mathrm{Ga} / \mathrm{P}<1)$ thin films spectra present a mixed feature between those of the two crystallized standards. Indeed, the intensity and the width of the white line $A$, attribuated to $1 \mathrm{~s} \rightarrow 4 \mathrm{p}$ electron transitions, are intermediate to those of $\mathrm{\alpha GaPO}_{4}$ and $\mathrm{GaOOH}$. Futhermore, the second feature B, rather due to multiple scattering effects and present as a peak for $\alpha \mathrm{GaPO}_{4}$, exists as a shoulder for $\mathrm{Ga} / \mathrm{P}<1$ films. These indicate the existence of mixed surroundings of the $\mathrm{Ga}$ atoms in the $\mathrm{Ga} / \mathrm{P}=<1$ deposits, constituted of tetrahedra $\mathrm{GaO}_{4}$ and octahedra $\mathrm{GaO}_{6}$ as encountered in $\mathrm{BGa}_{2} \mathrm{O}_{3}$. In the case of $\mathrm{Ga}$-rich deposits $(\mathrm{Ga} / \mathrm{P}>1$ ), the shape of the $\mathrm{Ga}$ K-edge XANES curves, Figure 2, is closer to that of $\mathrm{GaOOH}$ than that of $\alpha \mathrm{GaPO} 4$. Indeed, in comparison with thin films enriched in phosphorus, we note, for these Ga-rich films, a broadening and an increase of the intensity of the $\mathrm{A}$ white line and an absence of the $\mathrm{B}$ feature characteristic of $\mathrm{GaO}_{4}$ units. Thus, it seems that $\mathrm{GaO} 6$ sites are predominant in this thin film.

These qualitative structural results have been completed by a Ga K-edge EXAFS analysis. Figure 3 shows the Fourier Transform moduli, uncorrected for phase shifts, of thin films of various compositions. The simulations of the Fourier filtered experimental signals have been performed using a mean-square fitting procedure. Backscattered phases and amplitudes were 
extracted from the EXAFS signal of $\alpha_{\mathrm{GaPO}} 4$ compound. The fit results obtained for the raw thin films are given in Table 1 . The coordination number obtained for the P-rich deposit $(\mathrm{N}=5)$ is similar to the $\mathrm{\beta G}_{2} \mathrm{O}_{3}$ one in agreement with mixed surroundings of $\mathrm{Ga}$ atoms pointed out by XANES analysis. For Ga-rich deposits $(\mathrm{Ga} / \mathrm{P}>1)$, the EXAFS results show that Ga atoms are only located in distorted octahedral sites $(\mathrm{N}=6)$. The $\mathrm{Ga}-\mathrm{O}$ bond lengths obtained here are shorter than those encounterd in the $\mathrm{Ga}$ sites of $\beta \mathrm{Ga}_{2} \mathrm{O}_{3}(\mathrm{Ga}-\mathrm{O} \sim 1.91 \AA)$ and $\mathrm{GaOOH}(\mathrm{Ga}-\mathrm{O} \sim 1.99 \AA)$. So, to check the validity of our treatment,

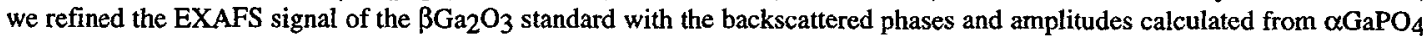
EXAFS signal. We obtained for the coordination number $N=4.7$ instead of $N=5$ (Table 1), and for the bond length $R=1.87 \AA$ instead of $R=1.91 \AA$. This is certainly due to the use of the tetrahedral environment, $\alpha \mathrm{GaPO}_{4}$, as a reference leading to an imperfect modelling of the octahedral one (problem of phase transferability). However, the distance evolution is consistent with those of coordination numbers and Debye-Waller factors and the XANES results. The weak intensity of the second FT peak for the $\mathrm{Ga} / \mathrm{P}=3.6$ deposit prevented us from making correct adjustments. The shape of the backscattered amplitudes extracted from this peak characterizes the $\mathrm{Ga}$ atoms resulting from $[\mathrm{O}-\mathrm{Ga}-\mathrm{O}-\mathrm{Ga}]_{\mathrm{n}}$ chains.

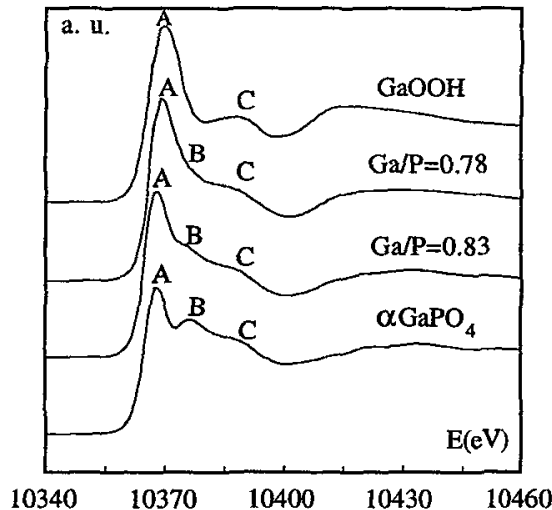

Figure 1 : Ga K-edge normalized XANES spectra of a P-rich film and crystallized references .

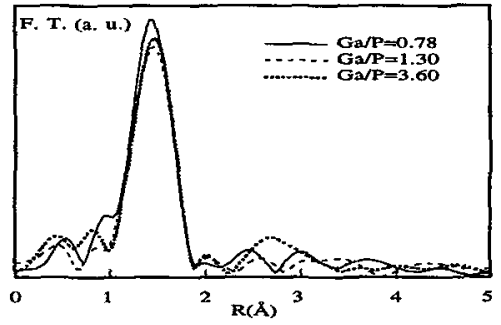

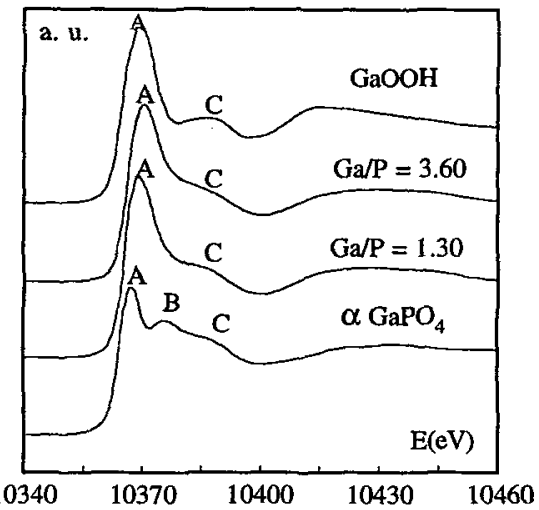

Figure 2 : Ga K-edge normalized XANES spectra of a Ga-rich deposit and crystalline phases.

\begin{tabular}{|c|c|c|c|c|c|}
\hline $\mathrm{Ga} / \mathrm{P}$ & $\mathrm{O}_{\mathrm{th}} / \mathrm{O}_{\exp }$ & $\mathrm{N}_{\mathrm{Ga}-\mathrm{O}}$ & $\mathrm{R}(\AA)$ & $\sigma(\AA)$ & $\mathrm{RF}^{*}$ \\
\hline 0.78 & 0.81 & 4.9 & $1.83(1)$ & $0.045(3)$ & $7.710^{-3}$ \\
0.83 & 0.82 & 5.0 & $1.84(1)$ & $0.059(3)$ & $4.510^{-3}$ \\
1.30 & 0.82 & 6.1 & $1.88(1)$ & $0.077(3)$ & $8.910^{-3}$ \\
3.60 & 0.76 & 5.9 & $1.89(1)$ & $0.075(3)$ & $2.010^{-2}$ \\
\hline$\beta \mathrm{Ga}_{2} \mathrm{O}_{3}$ & & 4.7 & $1.87(1)$ & $0.095(4)$ & $1.610^{-2}$ \\
\hline
\end{tabular}

*RF : reability factor

Figure 3 : Fourier Transform moduli, uncorrected for phase shifts, obtained from $\mathrm{Ga}$ K-edge oscillations of several deposits. Table 1 : Fit results for thin films and for the standard $\mathrm{\beta Ga}_{2} \mathrm{O}_{3}$.

\section{OXYGEN ENVIRONMENT}

We completed the structural characterization of the gallium phosphate thin films by a qualitative XANES study of the oxygen surroundings. Oxygen K-edge (532 eV) XANES spectra of deposits of various compositions (Figure 4) are directly compared with those recorded for crystalline phases; cristobalite $\mathrm{GaPO}_{4}$ (oxygen atoms are dicoordinated, $\mathrm{Ga}-\mathrm{O}-\mathrm{P}$ [4]) and $\mathrm{BGa} 2 \mathrm{O}_{3}(\mathrm{O}$ atoms are both tri and tetra-coordinated with Ga atoms [5]). The thin films XANES spectra are rather similar and are close to the one registered for the reference cristobalite $\mathrm{GaPO}_{4}$ (Figure 4), with dicoordinated oxygen atoms. Nevertheless, we can note between the cristobalite $\mathrm{GaPO}_{4}$ and the thin films spectra, a broadening and an intensity reduction of the different features due to classical disorder effects of amorphous materials.

The high similitude of the $\mathrm{Ga} / \mathrm{P}=1.35$ spectrum with that of cristobalite $\mathrm{GaPO}_{4}$ (Figures 4 and 5), seems to indicate that the local structure around $\mathrm{O}$ atoms in the deposit is based on that encountered in this crystallized phase (Ga-O-P). When the composition of the layer gets richer in phosphorus $(\mathrm{Ga} / \mathrm{P}=0.67)$ or in gallium $(\mathrm{Ga} / \mathrm{P}=2.87)$, the intensity of the resonance 
structure around $541.5 \mathrm{eV}$ (Figures 4 and 5) decreases and appears like a shoulder. This evolution may be related to local range order modifications due to the coexistence of chains of Ga-O-P and P-O-P types for Ga/P=0.67 and Ga-O-P and Ga-O-Ga type for $\mathrm{Ga} / \mathrm{P}=2.87$.

The oxygen surroundings characterization has been completed by an $O$ is core-level $X$-ray photoelectron spectroscopy (XPS) study. In order to get structural information from XPS spectra, the best solution is to compare the binding energy for unknown samples with that from well known standard compounds. To decompose the $G a / P=0.70$ XPS spectrum, two components were needed. The one centered at $531.6 \mathrm{eV}$ is attribuated to $\mathrm{O}$ atoms bridging a $\mathrm{Ga}$ and a $\mathrm{P}$ atom ( $\mathrm{P}-\mathrm{O}-\mathrm{Ga}$ ), while the smaller intensity component at higher energy $(533.5 \mathrm{eV})$ corresponds to $\mathrm{O}$ atoms linked to two $\mathrm{P}$ ones (P-O-P). These results are in agreement with those given by Hollinger et al. [8] who, for a P-rich indium phosphate layer, used two gaussian functions to decompose the $O 1$ s core-level spectrum. The most intense peak, centered at $531.7 \mathrm{eV}$, has been attribuated to In$\mathrm{O}-\mathrm{P}$ linkages, while the less intense one at $533.7 \mathrm{eV}$ corresponds to $\mathrm{O}$ atoms linking two $\mathrm{P}$ atoms.

For the $\mathrm{Ga} / \mathrm{P}=1.15$ deposit, the assymetric XPS spectra has been decomposed with two components. The most intense one at $532.1 \mathrm{eV}$ is easily attribuated to $\mathrm{Ga}-\mathrm{O}-\mathrm{P}$ chains. The second one, at lower binding energy ( $529.7 \mathrm{eV}$ ), is probably due to non bridging oxygen ( $\mathrm{Ga}-\mathrm{O}$ ). Finally, to decompose the XPS spectrum of the $\mathrm{Ga} / \mathrm{P}=2.70$ thin film, only one gaussian function was necessary centered at $531.8 \mathrm{eV}$. This binding energy may indicate the existence of mixed surroundings of the dicoordinated oxygen atoms: $\mathrm{Ga}-\mathrm{O}-\mathrm{P}$ and $\mathrm{Ga}-\mathrm{O}-\mathrm{Ga}$. Indeed, the $\mathrm{Ga}$ atoms being less electronegative $(\chi \mathrm{Ga}=1.82)$ than the $\mathrm{P}$ ones $(\chi \mathrm{P}=2.1)$, the Is core-level binding energy of $\mathrm{O}$ atoms bridging two $\mathrm{Ga}$ atoms ( $\mathrm{Ga}-\mathrm{O}-\mathrm{Ga}$ ) should be situated at lower energy than that due to $\mathrm{O}$ atoms linked to a $\mathrm{P}$ and a Ga atom (Ga-O-P $\sim 532.3 \mathrm{eV}$ ). This attribution is concordant with the Ga K-edge EXAFS results, which has qualitatively pointed out the existence of $\mathrm{Ga}$ atoms in second coordination sphere of gallium, corresponding to Ga-O-Ga bridges, for a deposit of $\mathrm{Ga} / \mathrm{P}=3.6$ composition.

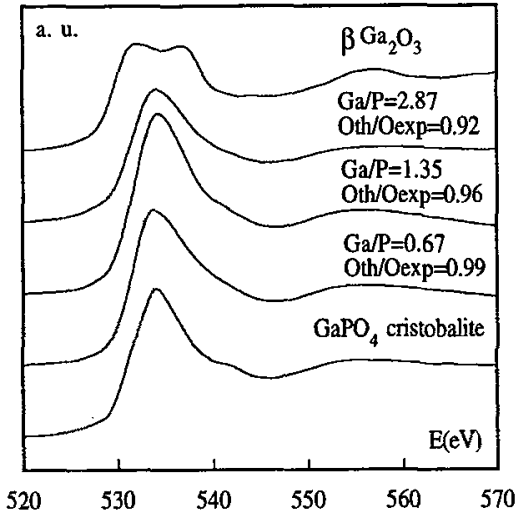

Figure 4: O K-edge normalized XANES spectra.

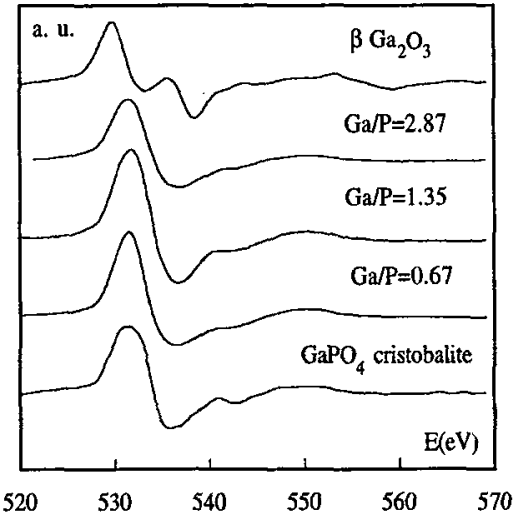

Figure 5: O K-edge derivative absorption curves .

\section{CONCLUSION}

Function of their chemical composition, the gallium phosphate thin films deposited on Si substrate present characteristic local structures. For P-rich deposits, the $\mathrm{Ga}$ atoms are located in mixed surroundings constituted of $\mathrm{GaO}_{4}$ and $\mathrm{GaO}_{6}$ units. The dicoordinated $\mathrm{O}$ atoms form two types of linkages, Ga-O-P and P-O-P. When the composition is slightly enriched in Ga content $(1.1<\mathrm{Ga} / \mathrm{P} \leq 2)$, the $\mathrm{GaO}_{6}$ octahedra are mainly linked through Ga-O-P bridges. However, some non-bridging oxygen atoms are present. For $\mathrm{Ga} / \mathrm{P}>2$ compositions, the $\mathrm{Ga}$ atoms are in octahedral sites. The dicoordinated $\mathrm{O}$ atoms are shared between $[\mathrm{Ga}-\mathrm{O}-\mathrm{Ga}-\mathrm{O}]_{n}$ chains and $[\mathrm{Ga}-\mathrm{O}-\mathrm{P}-\mathrm{O}-\mathrm{Ga}]_{n}$ ones, of which proportions depend on the composition.

\section{References}

[1] Détaint J., Schwartzel J., Joly C., Capelle B., Zarka A., Zheng Y., Toudic Y. and Philippot E., 6th E.F.T.F., (1992) 223.

[2] Viguié J.C.and Spitz J., J. Electrochem. Soc., 122 (1975) 585.

[3] Tourtin F., Daviero S., Ibanez A., Haidoux A., Avinens C. and Philippot E., J. Non-Cryst. Solids, 187 (1995)435.

[4] Mooney R.C.L., Acta Cryst., 9 (1956) 728.

[5] Geller S., J. Chem. Phys., 33 (1960) 676.

[6] Goiffon A., Bayle G., Astier R., Jumas J.C., Maurin M. and Philippot E., Revue Chimie Min., 20 (1983) 318.

[7] Pye M.F., Birtill J.J. and Dickens P.G., Acta Cryst., 33 (1977) 3224.

[8] Hollinger G., Joseph J., Robach Y., Bergignat E., Vicktorovith P. and Froment M., J. Vac. Sci. Technol., B5 (1987) 1108. 